phys. stat. sol. (a) 192, No. 2, 286-291 (2002)

\title{
Deep Ultraviolet AIGaInN-Based Light-Emitting Diodes on Si(111) and Sapphire
}

\author{
G. Kipshidze (a), V. Kuryatkov (a), B. Borisov (a), S. Nikishin ${ }^{1}$ ) (a), \\ M. Holtz (b), S. N. G. Chu (c), and H. Temkin (a) \\ (a) Department of Electrical Engineering, Texas Tech University, Lubbock, TX 79401, USA \\ (b) Department of Physics, Texas Tech University, Lubbock, TX 79401, USA \\ (c) Agere Systemss, Murray Hill, NJ 07974, USA
}

(Received February 18, 2002; accepted March 20, 2002)

PACS: 68.37.Lp; 78.60.Fi; 78.60.Hk; 81.15.Hi; 85.60.Jb

\begin{abstract}
Ultraviolet light-emitting diodes (LEDs) with emission wavelength as short as $280 \mathrm{~nm}$, grown by gas source molecular beam epitaxy with ammonia, are described. The typical multi-quantum well (MQW) structure LED consists of an AlN buffer layer deposited on Si(111) or sapphire, followed by a $(\mathrm{Al}) \mathrm{GaN}$ buffer layer and two superlattice structures, $\mathrm{n}$ - and p-type, with the MQW active region placed between them. Room temperature Hall measurements of $n$ - and p-type AlN/AlGaInN superlattice structures show average hole concentrations of $1 \times 10^{18} \mathrm{~cm}^{-3}$, with mobility of $3-4 \mathrm{~cm}^{2} / \mathrm{Vs}$, and electron concentrations of $3 \times 10^{19} \mathrm{~cm}^{-3}$, with mobility of $10-20 \mathrm{~cm}^{2} / \mathrm{Vs}$. Room temperature electroluminescence spectra of mesa-etched devices show predominant emission at $280 \mathrm{~nm}$.
\end{abstract}

Introduction There has been considerable recent interest in the preparation of ultraviolet light-emitting diodes (LEDs) based on alloys of AlGaInN [1-10]. Devices with emission wavelength between 340 and $280 \mathrm{~nm}$ would lead to a number of new applications, from fluorescence excitation to data storage. Despite recent progress, the preparation of light sources operating below $300 \mathrm{~nm}$ is still very difficult. Limits on p-type doping of $\mathrm{AlGaN}$ with high $\mathrm{Al}$ concentration can be overcome to some extent by the use of AlGaN/GaN [11-13] and AlGaInN/AlGaInN [7, 8] superlattices (SLs), allowing for the preparation of LEDs with emission wavelength close to $300 \mathrm{~nm}$. Recently, we have shown that SLs based on AlN/AlGaInN, doped with $\mathrm{Mg}$, can be used to increase the effective bandgap of the p-type cladding layer, demonstrating a $280 \mathrm{~nm}$ LED [9, 10]. We discuss the electrical and optical properties of these SLs and point out the importance of active structure design and careful post-growth device fabrication.

Experimental Two types of devices were investigated. The first consists of three SLs: $\mathrm{n}$ - and p-type emitters and an undoped active region [9]. Short-period SLs of $\mathrm{Al}_{0.4} \mathrm{Ga}_{0.6} \mathrm{~N} / \mathrm{Ga}(\mathrm{In}) \mathrm{N}$, each containing 25 pairs of quantum wells uniformly doped with $\mathrm{Si}$ and $\mathrm{Mg}$, are used to achieve high $\mathrm{n}$ - and p-type doping levels, respectively. Hall measurements of the p-type SL consisting of $1 \mathrm{~nm}$ thick layers of GaN separated by $5 \mathrm{~nm}$ thick layers of $\mathrm{Al}_{0.4} \mathrm{Ga}_{0.6} \mathrm{~N}$ showed an average hole concentration of $4 \times 10^{17} \mathrm{~cm}^{-3}$ with a hole mobility of $7 \mathrm{~cm}^{2} / \mathrm{Vs}$. Hall measurements of the n-type SL showed an electron concentration of $2 \times 10^{18} \mathrm{~cm}^{-3}$, sufficient for LED applications. The active region

1) Corresponding author; Phone: 1806742 3530; Fax: 1806742 8061;

e-mail: sergey.nikishin@coe.ttu.edu 
of the LED consists of five wells of GaInN ( $\left.x_{\text {In }}<0.01 \%\right)$ sandwiched between $5 \mathrm{~nm}$ thick barriers of $\mathrm{Al}_{0.4} \mathrm{Ga}_{0.6} \mathrm{~N}$. By varying the well thickness from $3.2 \mathrm{~nm}$ down to $0.6 \mathrm{~nm}$, we found that the peak emission wavelength shifted from 370 to $325 \mathrm{~nm}$. The maximum intensity occurred for a well thickness of $\sim 1.0 \mathrm{~nm}$, with a peak position at $\sim 340 \mathrm{~nm}$. Addition of a small amount of In to the GaN well doubles the emission intensity. The second LED type was based on SLs of AIN/AlGaInN grown on Si(111) [9] and on sapphire [10]. The AIN barriers were $\sim 1.2 \mathrm{~nm}$ thick. The wells of AlGaInN, with an AlN content of $\sim 0.1$, were $\sim 0.5 \mathrm{~nm}$ thick. Each SL consisted of 150 barrier/well pairs. The p-type SL, doped with $\mathrm{Mg}$, had an average hole concentration of $1 \times 10^{18} \mathrm{~cm}^{-3}$ with a mobility of $3-4 \mathrm{~cm}^{2} / \mathrm{Vs}$, at room temperature. The doping level in the n-type SL, doped with $\mathrm{Si}$, was as high as $3 \times 10^{19} \mathrm{~cm}^{-3}$, with a mobility of $10-20 \mathrm{~cm}^{2} /$ Vs. The active region of this device consisted of five pairs of undoped AlN/AlGaInN SLs.

Two points should be made about epitaxial growth of these LED structures. First, the quality of the buffer layer is very important to the successful preparation of the device. This is illustrated in Fig. 1a. The transmission electron microscopy (TEM) cross-section shows the buffer layer grown on sapphire. Epitaxial growth is started with the nitridation of the substrate, followed by the deposition of $\mathrm{AlN}$, at $900{ }^{\circ} \mathrm{C}$. A $40 \mathrm{~nm}$ thick layer of AlN is then grown to produce a two-dimensional (2D) surface with Al polarity. A buffer layer of $\mathrm{GaN}$ or $\mathrm{AlGaN}$, with an $\mathrm{Al}$ content of 0.1 , is grown next, under $2 \mathrm{D}$ conditions. As shown in Fig. 1a, the majority of dislocations are confined to the buffer and the SL-based device structure is grown with a drastically lower defect density. Low defect density is expected in SL structures with narrow wells and barriers [14]. In the absence of a buffer layer, the high quality of short-period SLs cannot be assured. Second, even when the buffer layer is present, high-quality growth of the SL requires a high quality of control. Gas source molecular beam epitaxy provides excellent control over the growth rate and fluxes [15] needed to produce sub-nanometer SLs. This is shown in the TEM cross-section of Fig. 1b illustrating the AlN/AlGaInN SLs of the LED structure. The wells and barriers appear to be uniform and continuous, even at sub-nanometer dimensions.

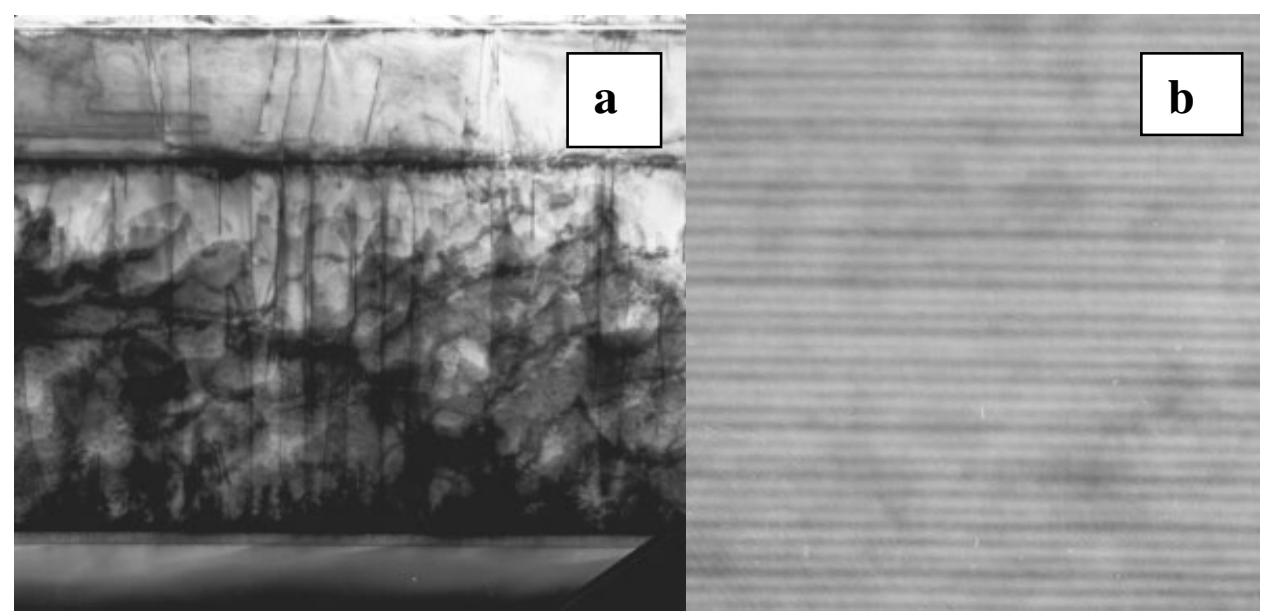

Fig. 1. TEM cross-section images of a) the entire device structure and b) its upper part consisting of AlN/AlGaInN SLs only 


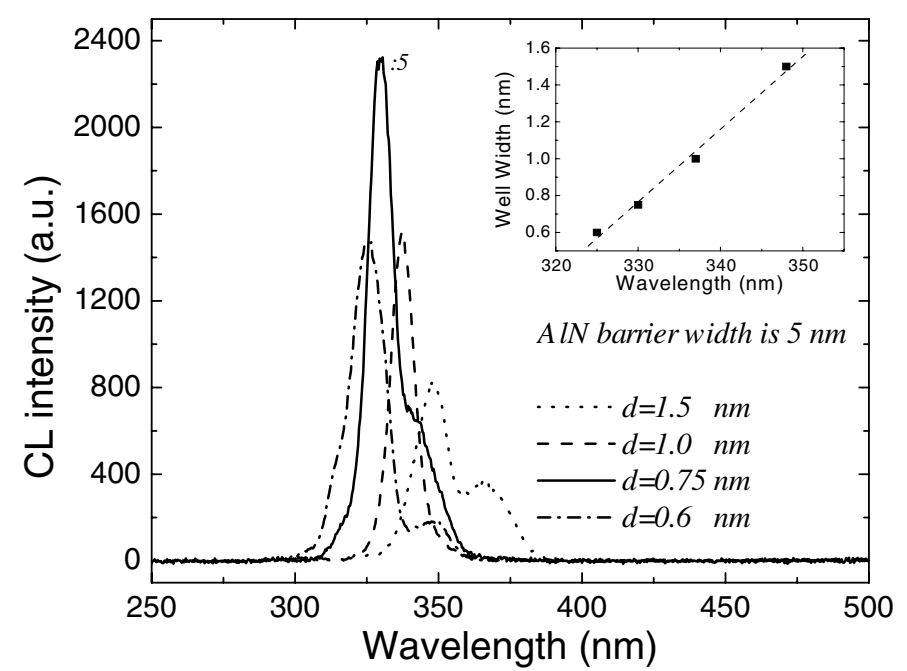

Fig. 2. Room temperature CL spectra of $\mathrm{Al}_{0.4} \mathrm{Ga}_{0.6} \mathrm{~N} / \mathrm{GaN}$ MQWs. The inset shows the wavelength dependence on the well thickness

Figure 2 shows cathodoluminescence (CL) spectra of the active layer multi-quantum well (MQW) designed for $340 \mathrm{~nm}$ operation, with the active region based on $\mathrm{GaInN}$ wells and $\mathrm{Al}_{0.4} \mathrm{Ga}_{0.6} \mathrm{~N}$ barriers. In the set of samples shown, the well thickness was changed from 1.5 to $0.6 \mathrm{~nm}$. This has two effects. There is a blue shift with decreasing well thickness, from 350 to $325 \mathrm{~nm}$. The shift, illustrated in detail in the inset of Fig. 2, is linear and controllable. There is also a strong, and very reproducible, dependence of the CL intensity on the well thickness. It has a pronounced maximum at a well width of $\sim 0.75 \mathrm{~nm}$ (the corresponding spectrum in Fig. 2 is divided by a factor of 5). The optimum CL spectrum is quite narrow, with a full width at half maximum (FWHM) of less than $11 \mathrm{~nm}$. Addition of a small amount of In, less than $0.01 \%$, to the GaN wells increases the overall luminescence intensity by factors of $3-5$, without changing the well thickness dependence. The double-peak emission often observed in the spectra of these wells is believed to arise from growth procedure artifacts. For instance, the $\mathrm{Al}$ and $\mathrm{Ga}$ fluxes increase when the shutters are opened and the resulting flux transients change the composition of the well. This may be less important in the growth of narrower wells, which take only 3-5 s to grow. Their spectra show slight broadening, FWHM $\sim 15-20 \mathrm{~nm}$, and weak substructure that is not resolved at room temperature.

Figure 3 shows room temperature electroluminescence (EL) spectra from the $334 \mathrm{~nm}$ LED grown on $\mathrm{Si}(111)$. The EL spectrum was obtained under DC bias, with a forward current of $10 \mathrm{~mA}$ and a forward voltage $V_{\mathrm{f}} \sim 15 \mathrm{~V}$. The LED emits at $334 \mathrm{~nm}$ in a narrow, $\sim 12 \mathrm{~nm}$ FWHM, and symmetric peak. We did not observe any defect emission at longer wavelengths. Similar performance was obtained from $340 \mathrm{~nm}$ LEDs grown on sapphire substrates.

LEDs designed for $280 \mathrm{~nm}$ operation were grown on both $\mathrm{Si}(111)$ and sapphire substrates. Figure 4 shows EL results obtained for AIN/AlGaInN LEDs grown on sapphire. The short-wavelength devices exhibit complex behavior, strongly influenced by the design of the active region and the device fabrication procedure. Preliminary mea- 


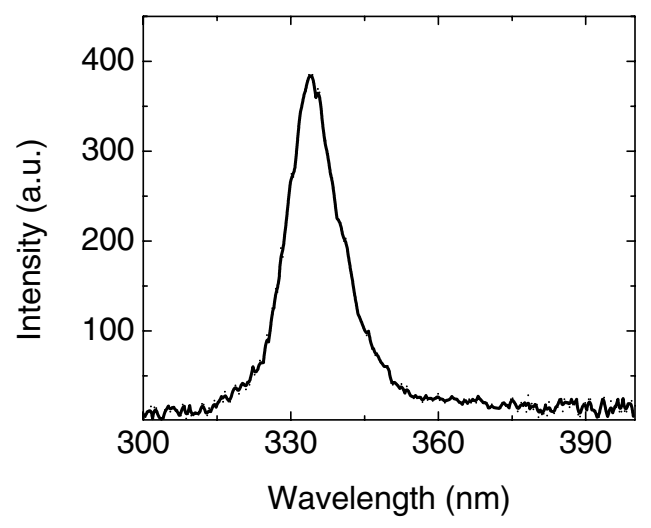

Fig. 3. Room temperature CW EL obtained from an $\mathrm{Al}_{0.4} \mathrm{Ga}_{0.6} \mathrm{~N} / \mathrm{GaN}$ MQW LED grown on $\mathrm{Si}(111)$

surements were carried out on devices prepared by depositing $\mathrm{Ni}$ contact dots with two different diameters, 70 and $500 \mu \mathrm{m}$, on the p-type surface. The large diameter contact serves as a cathode. Light is collected with a UV-transparent fiber from the edge of the small dot, the p-type contact, and analyzed with a spectrometer. No light emission was observed around the large contact dot. EL spectra of LEDs grown with an abrupt n- to p-type transition exhibit only the $\sim 330 \mathrm{~nm}$ emission (not shown in Fig. 4) which we associate with recombination in n-type SLs. Figure 4a shows the EL spectrum obtained for a structure grown without Si doping in the n-type SL. It shows emission at $280 \mathrm{~nm}$, in addition to the $\sim 330 \mathrm{~nm}$ peak. Light emission is visible at forward DC currents as low as $2 \mathrm{~mA}$ (at $V_{\mathrm{f}} \sim 10 \mathrm{~V}$ ) and the $280 \mathrm{~nm}$ peak increases rapidly with forward current. EL spectra of a structure that incorporates five undoped well/barrier pairs between SLs doped with $\mathrm{Si}$ and $\mathrm{Mg}$ also show two peaks, at 280 and $330 \mathrm{~nm}$, not shown in Fig. 4. When driven with pulsed current, up to $350 \mathrm{~mA}$, the intensity of the $280 \mathrm{~nm}$ peak dominates. The spectrum of Fig. $4 \mathrm{~b}$ was obtained on the same structure, with five undoped barrier/well pairs, after $0.5-0.6 \mu \mathrm{m}$ deep mesas were etched around contact dots, through the $\mathrm{p}-\mathrm{n}$ junction that is estimated to be $\sim 0.3 \mu \mathrm{m}$ deep. The mesas were plasma etched with $\mathrm{Cl}$ chemistry using $\mathrm{Ni}$ contacts as masks. EL spectra of the etched structure show predominant emission at $280 \mathrm{~nm}$, with the $330 \mathrm{~nm}$ line reduced to a shoulder, at all currents.

A simple configuration of metal dots on the p-type surface appears to result in large leakage current. This is not surprising given the nature of SL structures that provide high in-plane conductance. The relative intensity of the $330 \mathrm{~nm}$ peak correlates with leakage current as well as the presence of $\mathrm{Si}$ at the junction. Once the $\mathrm{Si}$ is pushed

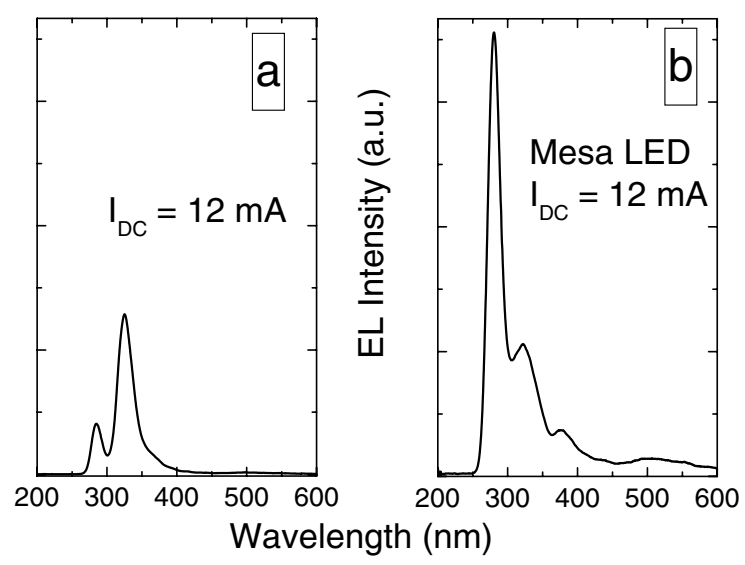

Fig. 4. Room temperature EL spectra: a) LED structure with n-type SL grown without Si doping; b) mesa structure LED with an active region formed by five undoped well/barrier pairs. Intensities are on the same scale 
back, by incorporation of an undoped active region, the competing near-bandedge emission at $280 \mathrm{~nm}$ takes over. In such a structure mesa etching is effective in eliminating lateral conduction. Formation of an active region with better carrier confinement is needed in order to minimize competing radiative recombination. Such an active region can be formed, without affecting the emission wavelength, by increasing the effective bandgap of the $\mathrm{n}$ - and p-type SLs or by the introduction of thicker AlN barriers around the undoped recombination region.

Discussion We describe the successful preparation of LEDs operating between 280 and $340 \mathrm{~nm}$. These devices are based on tunneling SL structures. The use of doped SLs was proposed in order to bypass the acceptor incorporation limits in AlGaN [16]. Experimentally, average hole concentrations at least 10 times higher than that achieved in a uniform layer appear possible [11-13]. The concept was used by Nakamura et al. [17] in order to reduce series resistance of laser structures and was applied recently to UV LEDs [1-10]. Further refinements through modulation doping appear feasible [18]. Replacement of a difficult to dope uniform layer with a SL in which the effective hole density is determined only by its geometry is crucial to the successful preparation of UV light-emitting devices. The work described here shows that the idea can also be applied to SLs with AIN barriers.

Conclusion We describe LEDs based on n- and p-type SLs of AlGaN/GaInN and AlN/ AlGaInN with emission wavelengths between 340 and $280 \mathrm{~nm}$. These devices are grown on $\mathrm{Si}(111)$ and sapphire substrates using gas source molecular beam epitaxy with ammonia.

Acknowledgements This work was supported by DARPA (under a contract monitored by Dr. J. Carrano), SBCCOM, NSF (grant ECS-0070240), CRDF (grant RE1-2217), NATO Science for Peace Program (grant SfP-974505) and the J. F. Maddox Foundation. The authors thank Riber Inc. for an extended cold lip Al effusion cell used for epitaxy in this work.

\section{References}

[1] K. Tadamoto, H. Okagawa, Y. Ohuchi, T. Tsunekawa, Y. Imada, M. Kato, and T. Taguchi, Jpn. J. Appl. Phys. 40, L583 (2001).

[2] T. Nishida, H. Saito, and N. KobaYashi, Appl. Phys. Lett. 78, 3927 (2001).

[3] T. Nishida, H. Saito, and N. Kobayashi, Appl. Phys. Lett. 79, 711 (2001).

[4] A. Kinoshita, H. Hirayama, M. Ainoya, Y. Aoyagi, and A. Hirata, Appl. Phys. Lett. 77, 175 (2000).

[5] H. Hirayama, A. Kinoshita, and Y. Aoyagi, RIKEN Review 33, 28 (2001).

[6] H. Hirayama, A. Kinoshita, M. Ainoya, A. Hirata, and Y. Aoyagi, phys. stat. sol. (a) 188, 83 (2001).

[7] M. A. Khan, V. Adivarahan, J. P. Zhang, C. Chen, E. Kuokstis, A. Chitnis, M. Shatalov, J. Wei, and G. Simin, Jpn. J. Appl. Phys. 40, L1308 (2001).

[8] V. Adivarahan, A. Chitnis, J. P. Zhang, M. Shatalov, J. W. Yang, G. Simin, and M. A. Khan, Appl. Phys. Lett. 79, 4240 (2001).

[9] G. Kipshidze, V. Kuryatkov, B. Borisov, M. Holtz, S. Nikishin, and H. Temkin, Appl. Phys. Lett. 80, 3682 (2002).

[10] G. Kipshidze, V. Kuryatkov, B. Borisov, K. Zhu, M. Holtz, S. Nikishin, and H. Temkin, submitted for publication in Appl. Phys. Lett.

[11] P. Kozodoy, M. Hansen, S. P. DenBaars, and U. K. Mishra, Appl. Phys. Lett. 74, 3681 (1999).

[12] K. Kumakura and N. Kobayashi, Jpn. J. Appl. Phys. 38, L1012 (1999). 
[13] K. Kumakura, T. Makimoto, and N. Kobayashi, Jpn. J. Appl. Phys. 39, 2428 (1999).

[14] A. D. Bykhovski, B. L. Gelmont, and M. S. Shur, J. Appl. Phys. 78, 3691 (1995).

[15] M. B. Panish and H. Temkin, Gas Source Molecular Beam Epitaxy, Springer-Verlag, Berlin 1993.

[16] E. F. Schubert, W. Grieshaber, and I. D. Goepfert, Appl. Phys. Lett. 69, 3737 (1996).

[17] S. Nakamura, M. Senoh, S. Nagahama, N. Iwasa, T. Yamada, T. Matsushita, H. Kiyoku, Y. Sugimoto, T. Kozaki, H. Umemoto, M. SAno, and K. Сносно, Jpn. J. Appl. Phys. 36, L1568 (1997).

[18] E. Waldron, J. W. Graff, and E. F. Schubert, Appl. Phys. Lett. 79, 2737 (2001). 